NORMAS. REVISTA DE ESTUDIOS LINGÜÍSTICOS HISPÁNICOS, N. 3, 2013, PP. 77-97, ISSN: $2174-7245$

(RECIBIDO: 8-09-2013. EvaluAdo: 15-09-2013. ACEPTADO: 20-09-2013)

https://doi.org/10.7203/Normas.3.4674

\title{
LENGUA DE SEÑAS ARGENTINA (LSA) Y ESPAÑOL EN LA ALFABETIZACIÓN DE ALUMNOS SORDOS. APORTES PARA LA PRÁCTICA EDUCATIVA
}

\author{
ARGENTINA SIGN LANGUAGE (LSA) AND SPANISH IN THE LITERACY OF DEAF \\ STUDENTS. CONTRIBUTIONS TO EDUCATIONAL PRACTICE
}

\author{
MARÍA EUGENIA LAPENDA \\ Dirección de Educación Especial. Provincia de Buenos Aires \\ Instituto del Profesorado en Educación Especial. Ciudad Autónoma de Buenos Aires
}

RESUMEN:

En el desarrollo de un modelo educativo Intercultural y bi(pluri)lingüe (lengua de señas argentina -LSA- y español como lengua segunda) y otras lenguas extranjeras, lenguas muy disímiles constituyen la realidad lingüística del escenario educativo. El objetivo de este artículo es plantear algunas reflexiones acerca de la relación entre ellas en el proceso de alfabetización de alumnos sordos.

PALABRAS ClAVE: estudiantes sordos; bilingüismo; lengua de señas; español como lengua segunda.

\section{ABSTRACT:}

In the development of an intercultural and bilingual educational model (LSA-spanish as a second language) and other foreign languages, different languages constitute the linguistic reality of the educational context. The aim of this paper is to present some reflections about the relationship between them in the literacy process of deaf students.

KEY WORDS: deaf students; bilingualism; sign language; spanish as a second language. 


\section{LA RELACIÓN ENTRE LENGUAS EN LA EDUCACIÓN BILINGÜE}

Las lenguas no hacen más que manifestar las múltiples posibilidades y riquezas del lenguaje humano. La acumulación de evidencia a favor de leyes linguiísticas abstractas, presentes en las lenguas más diversas, es uno de los mayores logros de la lingüística. Más allá de sus diferencias, todas las lenguas, están regidas por las mismas leyes. El interés de la lingüística es, entonces, la facultad del lenguaje y no las lenguas en sí, dado que en realidad las lenguas permiten conocer esta compleja facultad (Torrego, 1998).

Este artículo versará sobre la educación bilingüe. Precisamente en este campo, se constata la relación de transferencia mutua que se establece cuando varias lenguas interactúan. Tal fenómeno continúa siendo objeto de investigación en los procesos de adquisición de lenguas segundas-extranjeras en la actualidad, en especial, por el interés en el diseño de nuevas propuestas didácticas (Salazar, 2006; Delmastro, 2005; Mitchell y Myles, 2004; Durgunoglu, 2002; Murphy, 2002; Kerper-Mora, 2000; Krashen 1996, 1983, 1982; Ellis, 1994, Odlin, 1989; Gass, 1984).

La hipótesis de la transferencia se apoya en la convicción de que existen procesos mentales subyacentes que son comunes en el proceso de adquisición de la lengua 1 (L1) con los del aprendizaje de una segunda lengua o lengua extranjera (L2). Aunque los aspectos de las distintas lenguas están claramente separados, hay una competencia cognitivo-académica subyacente que es común entre ellas. Hay que superar, entonces, el concepto de una separación rígida y completa de las lenguas en el aprendizaje, separación que no es tal para el conocimiento cognitivo, conceptual ni lingüístico (Cummins, 2004).

Los conocimientos adquiridos conforman estructuras mentales (esquemas) y cumplen luego un rol importantísimo en el procesamiento de información nueva (Anderson, 1984 citado por Salazar, 2006). Es necesario así que se activen los esquemas ya existentes que estén relacionados con un nuevo contenido (Widmayer, 2000, citado por Salazar, 2006). En este sentido, Cummins (2004) acentúa la necesidad de implementar estrategias que sirvan para activar el conocimiento previo en L1 durante el proceso de aprendizaje de cualquier aspecto de la lengua meta.

El tipo de enseñanza basada en esta posibilidad aumenta la comprensión de los conceptos lingüísticos; se transfieren de una lengua a otra las especificidades de cada una, los elementos conceptuales y las estrategias de aprendizaje.

\subsection{Un modelo educativo para estudiantes sordos: LS-lengua mayoritaria}

En relación con un modelo de educación bilingüe para alumnos sordos, algunos autores, no obstante, plantean dificultades relativas a la transferencia lingüística.

En la actualidad, han cobrado ya amplia difusión las diferencias básicas entre estas lenguas de diferente modalidad. A grandes rasgos, se trata de lenguas que se distinguen en 
el modo de estructurar los significantes ${ }^{1}$, con diferencias en el orden canónico (por ejemplo, encontramos un orden canónico sujeto - verbo - objeto para el español y sujeto - objeto verbo en la $\mathrm{LSA}^{2}$ ), en los procesos morfológicos.

Por estas razones fundamentalmente, ciertos autores objetan algunos puntos importantes de un modelo de bilingüismo aplicado a sordos. Para Mayer y Akamatsu (1999), que la L1 (LS) sea ágrafa ${ }^{3}$ constituye un impedimento para transferir los conocimientos sobre escritura de L1 a L2 (lengua mayoritaria). Otro argumento esgrimido se relaciona con la falta de conocimiento lingüístico de la L2, que en los oyentes suele desarrollarse en una primera etapa a través de la oralidad y que en alumnos sordos no siempre puede ser previo a la lectura y la escritura. En la misma línea, Andrews y Mason (1991) sostienen que la forma del lenguaje de señas es estructuralmente diferente del lenguaje oral.

Sin embargo, en el desarrollo de un nuevo paradigma educativo que integra la LS en la propuesta curricular se destaca el papel de la transferencia para la dupla LS- lenguas orales, como fuera señalado ya por Baker (1993); Svartholm (1993); Berent (1996); Berent y Clymer (2007); Fernández Viader (1996 y 1997); Padden y Ramsey (2000); Hoffmeister, Strong y Prinz (1998 y 2000); Herrera (2003); Neese Bailes et al. (2009); Petitto (2000); Rusell y Lapenda (2007); Wilbur (2000); Collier (1995); Heiling (1999); Volterra (1981); entre muchos otros. De hecho, es observable en las aulas cómo los alumnos sordos transfieren entre lenguas conocimientos de diversa clase. Y ello es, en gran parte, el corazón del paradigma.

Si la transferencia no fuera posible, no podríamos afirmar que los sujetos sordos son bilingües, ni tampoco construir un modelo de alfabetización en el que interviene la LS, ni hablar de los beneficios de la primera lengua para desarrollar, en nuestro caso, el español. Todos estos conceptos son centrales en los modelos de educación bilingüe para alumnos sordos. En este sentido, Petitto y Holowka (2000) demuestran que la exposición simultánea a dos lenguas, aún de distinta modalidad, no produce confusión ni retraso en el desarrollo del lenguaje. Lo mismo concluyen Volterra et al. (1979, 1982); Schelesinger y Meadow (1972). Por el contrario, encuentran que su presencia es favorecedora del desarrollo de la lengua mayoritaria.

Frente a estos argumentos opuestos a la posibilidad de transferencia, existen otras perspectivas de la productiva relación entre la L1 y la L2 en vistas a una propuesta alfabetizadora. La posibilidad de transferir conocimiento de una lengua a otra no es un

\footnotetext{
${ }^{1}$ Los trabajos de Benoit Virole exploran la estructuración de los significantes en LS, definiendo que el rasgo estructurante es de naturaleza icónica. Esta lengua codifica lingüísticamente los elementos de significación construidos por la experiencia perceptiva propia de la sordera (Virole, 2004).

${ }^{2}$ Por cierto, en LSA no es el único orden en que aparecen los constituyentes de la oración ${ }^{2}$, hecho que ocurre en todas las lenguas.

${ }^{3}$ La LS no tiene escritura, más allá de la transcripción mediante el sistema de glosas, sistema que persigue fundamentalmente fines de estudio.
} 
fenómeno circunscripto a la estructura sintáctica ni constatable exclusivamente entre lenguas «estructuralmente cercanas». Configura una realidad más abarcativa con la que nos referirnos a la influencia de una lengua sobre otra; por lo tanto, resaltamos que, precisamente una educación bilingüe que se adapte a alumnos sordos tiene el desafío de concretar una construcción particular que se ajuste a las características de las lenguas en cuestión y a los accesos más fructíferos para los alumnos.

Precisamente Cummins $(2006)^{4}$ habría formulado que el conocimiento conceptual, metacognitivo y metalingüístico, así como otros elementos lingüísticos, sí pueden transferirse desde la LS al lenguaje mayoritario. También Paul (1998) enfatiza el rol de la LS para las tareas metacognitivas en un modelo bilingüe metalingüístico y metacognitivo. La participación de la L1 permite la transferencia de conocimientos lingüísticos, por ejemplo las clases de palabras, y el acceso a un diálogo en el cual se indaguen las ideas previas de los alumnos, se establezcan propósitos, tareas, estrategias y formas de abordar los textos, se planifique y reflexione sobre los procesos de lectura y escritura.

\section{LA ALFABETIZACIÓN DE ALUMNOS SORDOS}

\subsection{Problemas relativos a la alfabetización de alumnos sordos}

Distintas investigaciones describen bajos niveles de comprensión lectora y producción escrita en sujetos sordos (Torres Monreal y Santana, 2005; Lapenda, 2005; Pertusa y Viader, 2004; Gutiérrez Cáceres 2004; Massone et al. 2003; Marschark et al. 2002; Stockseth Danzak, 2002; Traxler, 2000; Kuntze, 1998; Paul, 1998 y 1998a; Allen, 1986; Domínguez, 1995 y 1999; Valmaseda Balanzategui y Gómez Monteverde, 1999; Fernández Viader y Pertusa, 1996).

Asimismo, son elocuentes al echar luz sobre qué tipo de enunciados causan más dificultad en la lectura, como el dominio de frases simples frente a la dificultad con formas complejas como las cláusulas relativas (Rodríguez, García y Torres, 1997, corroborado por Mies, 1998) y sobre conocimiento léxico que no se corresponde con un buen nivel lector (Paul, 1998; King y Quigley, 1985). Muchas veces, se observa en los lectores sordos un procesamiento de los enunciados de tipo parte a parte y una relación significadosignificante en el tratamiento de cada palabra individualmente (Lapenda, 2005).

Con respecto a la escritura, Cooper y Rosenstein (1996) encontraron que las estructuras de oraciones son más cortas, simples, rígidas, estereotipadas y que los sordos producen mayor cantidad de errores sintácticos que los oyentes. Otros estudios desarrollados por Quigley, Wilbur y Montanelli (1976), para el inglés, confirman

\footnotetext{
${ }^{4}$ Cummins, J. (2006, October 27), citado por Hermans et al. (2008).
} 
habilidades sintácticas promedio de los sordos de 18 años más bajas que el promedio de los oyentes de 8 años.

A las dificultades evidenciadas por los sujetos sordos se les atribuyen distintas hipótesis explicativas; entre ellas, se encuentran las que refieren obstáculos de adquisición de la lectura como producto de su incapacidad lingüística.

Siegel (2000) subraya que los alumnos sordos fracasan por su falta de conocimiento de una lengua en particular (la mayoritaria) y no, por las características propias de la sordera. Por su parte, la LS es muestra de la capacidad linguística de los sordos quienes «se han dado lenguas desde la edad media»y, por ello, deben ser suficientemente valoradas como testimonio de que la naturaleza humana es profundamente simbólica (Díaz, 1999). Además, el español que los niños oyentes adquieren mediante la oralidad en edades tempranas no es incorporado por los niños sordos por la sola exposición al participar en una comunidad de hablantes, ni tampoco por trabajar únicamente su aspecto estructural o aprender vocabulario y realizar variedad de ejercitaciones.

Los dispositivos de ayuda auditiva (entre los que se destacan actualmente los implantes cocleares) que colaboran en la habilitación de la audición y la oralidad en el grado máximo posible, no garantizan a todos por igual la oralidad ni tampoco la competencia en lengua escrita porque las lenguas son diferentes $\mathrm{y}$, al enfrentarse a la alfabetización, los niños se encuentran aún con un conocimiento previo incompleto, fragmentado. El hecho de que algunos sordos tengan buenas habilidades en lenguaje oral, pero limitadas habilidades en lectura, sugiere, además, que estas no resultan simplemente de deficiencias en el lenguaje oral, sino que pueden estar asociadas a deficiencias en los procesos cognitivos específicos implicados en la lectura, y ello no se debe a la hipoacusia (Herrera, 2005).

Por otra parte, si la suficiencia lectora se evalúa mediante comparaciones entre niños sordos y oyentes, incluso con pruebas construidas para evaluar a estos últimos, está claro que el rendimiento lector del primer grupo será menor que el del segundo.

Del mismo modo, si para el acceso a la escritura se resalta, para el español, la importancia de la fonología porque la lengua es transparente, pareciera establecerse que la peculiaridad de la lengua hace que solo pueda adquirirse con una participación imprescindible de la audición y del léxico auditivo. Esta visión deja a los niños sordos inhabilitados frente a esta característica y, entonces, ser oyente es la única posibilidad para alfabetizarse.

En este sentido, un método oral puro parece insistir en el nivel de la dificultad de los alumnos, el canal más afectado. No obstante, algunos de los estudiantes entre aquellos que emplean de forma más productiva su audición, se enfrentan a puntos críticos y deben afrontar enormes diferencias lingüísticas con los oyentes, situación que es perfectamente identificable en contextos de integración escolar.

Particularmente, planteamos que las descripciones de la situación de los alumnos sordos deben ser leídas en clave de revisión de la alternativa pedagógica. Si bien los 
modelos exclusivamente orales han demostrado no ser lo suficientemente eficientes y los modelos bilingües han alcanzado mundialmente mayor desarrollo, cotidianamente aún se experimenta no solo tensión entre ellos sino deudas en ambos. Algunas posiciones profesionales desestiman la lengua de señas porque la consideran contraproducente; existen propuestas en las que se incluyen a los niños sordos en los mismos procesos de enseñanza de los niños oyentes y muchas veces, por otra parte, se organiza una educación en LS sin resolución de la propuesta de enseñanza que ha de seguirse para el español.

\subsection{Relaciones LSA-español en la alfabetización}

Rodríguez Ortíz y Mora Roche (2008) confirman la contribución de la LS en la comprensión lectora de lenguas mayoritarias. Corroborados por Marschark et al. (2007), estos datos son análogos a aquellos obtenidos por Neese Bailes et al. (2009); Hoffmeister (2002); Padden y Ramsey (2000); Chamberlain y Mayberry (2000), manifestados para el caso del inglés.

Herrera (2003, citado por la autora en 2005) concluye que la habilidad léxica (signada) contribuye significativamente al éxito lector de los estudiantes sordos en su investigación en Chile. Por su parte, Strong y Prinz $(1997,2000)^{5}$ encuentran que los niños sordos de padres oyentes leían tan bien como los niños sordos de padres sordos si tenían habilidades avanzadas del ASL (Lengua de Señas Americana). Por ello, concluyen que una educación bilingüe ayuda a todos los niños sordos a mejorar su capacidad de lectura y escritura en inglés (Instituto de la Sordera, Chile). ${ }^{6}$

Según mencionan Gibson et al. (1997), los alumnos sordos suecos logran obtener similares resultados en lectura que sus pares oyentes, a partir de la implementación de la metodología bilingüe. Del mismo modo, Hamilton y Holzman (1989) concluyen mejor rendimiento en los sordos bilingües que en los monolingües.

Estos y otros hallazgos corroboran el beneficio de una educación que despliegue y enriquezca tanto la L1 como la L2 y ponga a disposición del español el conocimiento desarrollado mediante la LSA.

El modelo bilingüe que concebimos no significa únicamente que los alumnos son usuarios de la LSA y que se favorecen de la transferencia de conocimientos entre lenguas. Más aún, la LSA interviene muy activamente en el proceso de adquisición de la lengua escrita.

En este sentido, se plantean dos líneas fundamentales. En primer lugar, la presencia de la LSA no debe entenderse como una particularidad especial de esta por sí misma como para permitir que el niño lea y escriba por sus propios medios. En segundo lugar, adherimos a posiciones que señalan que se necesita además no solo una efectiva instrucción en una conclusión.

${ }^{5}$ Con anterioridad en este trabajo, se mencionaron otros autores que se inclinan por la misma

${ }^{6}$ Acceso: <http://www.institutodelasordera.cl/crims/aprendizaje\%20lectoescritura.pdf $>$. 
lengua minoritaria que promueva la competencia en dicha lengua, sino también una exposición adecuada a la L2 (ya sea en la escuela o en el entorno) para que pueda darse la transferencia de la competencia de la L1 a la L2, junto con una motivación adecuada para aprender (Cummins, 2004).

En general, históricamente con los alumnos sordos se ha procedido mediante enfoques de corte estructuralista o en el extremo, desestimando el trabajo con el código, enfatizando muchas veces la adquisición de vocabulario, lejos de abordar la lectura y escritura desde el punto de vista psicolingüístico y comunicativo.

Por lo expuesto hasta aquí, postulamos una perspectiva compleja que integre todos estos aspectos sin desestimar ninguno. Definimos una propuesta de alfabetización cuyos principios estructurales son la intervención de la LSA como L1 con un rol muy activo en el proceso de alfabetización y la presentación de la lengua escrita como fuente de apropiación de español a través de la lectura. Desde una perspectiva psicolingüística, y tratada como objeto autónomo, la adquisición de la lectura podrá ocurrir por vía visual, en un marco dado por un enfoque de enseñanza del español como lengua segunda centrado, además, en las prácticas del lenguaje que concretan los hablantes, lectores y escritores ${ }^{7}$.

Seguidamente procederemos a hacer una breve referencia sobre cada aspecto, resaltando las relaciones entre las lenguas involucradas en este modelo educativo.

Por un lado, Davies (1994) señala que muchos de los maestros creen que los niños sordos aprenderán la conexión entre la lengua de señas y la lengua escrita ellos solos. Expresiones del tipo «se lee por la LS», «se alfabetiza en LS», «se aprende a leer por la $\mathrm{LS}$ », «escriben por la LS» suelen redundar y manifestar desconcierto. No es que por hablar LSA, luego podrán leer y escribir en español en una suerte de transferencia inmediata. La cuestión no se simplifica en la conclusión de que la LSA es la que permite aprender a leer y escribir.

Por ello, en nuestro modelo, la LSA confluye con un abordaje de lengua segunda para el español en sentido metodológico ${ }^{8}$, homologando la situación de los niños sordos frente al español, a la de los sujetos que se acercan a aprender una lengua segundaextranjera. Los enfoques de lengua segunda permiten, en nuestro caso, distinguir una situación particular de desconocimiento de la lengua meta anterior al inicio de la alfabetización.

Adoptar esta perspectiva para el español nos permite identificar que este no está consolidado en los alumnos y que, por lo tanto, tiene que ofrecerse mediante una didáctica particular. Esta perspectiva colabora tanto con alumnos que son hablantes de LSA como con quienes no lo son. Podemos afirmar que es muy ordenadora para aquellos niños que utilizan en gran medida su audición, para quienes son educados únicamente en forma oral

\footnotetext{
${ }^{7}$ Algunos alumnos aprovecharán más sus posibilidades auditivas.

${ }^{8} \mathrm{Se}$ destaca el sentido metodológico adoptado para el español lengua 2 para distinguir de posiciones que solo atribuyen su lugar como L2 al orden de adquisición y/o presentación de las lenguas en la educación, después de LS como lengua 1.
}

NORMAS. REVISTA DE ESTUDIOS LINGÜÍSTICOS HISPÁNICOS, NÚMERO 3 (AÑO 2013): 
(ya que les permite superar las restricciones que les supone la audición que van desarrollando) e inclusive para aquellos que realizan su escolaridad con pares oyentes.

Una visión similar para sujetos sordos es sostenida por Berent y Clymer (2007); Evans (2004), Andrews et al. (1997); Paul (1993); Svartholm (1993); Langston y Maxwell (1988); Charrow (1981); Charrow y Fletcher (1974) ${ }^{9}$.

Otro aspecto primordial es la adquisición de la lengua escrita por medio del procesamiento visual. $\mathrm{Si}$, en nuestro caso, se valora la propiedad de la transparencia del español como un principio facilitador de la adquisición por razones de economía y, al mismo tiempo, al mirar que otras lenguas (las opacas) suponen otros procesos (dado que no hay correspondencia sistemática entre lo ortográfico y lo fonológico), estamos apreciando una facultad del lenguaje humano que sí es posible para niños sordos: la adquisición por vía visual.

Igualmente, la fonología, entendida como representación interna de las palabras, necesita construirse y, como referiremos más adelante, puede hacerse de otros modos, hasta con un empleo efectivo de la audición, cuando sea posible. También es importante señalar que se observa frecuentemente cómo a partir de la escritura y de la LSA, puede significarse una señal acústica, que hasta ese momento, estaba desprovista de significado.

\subsubsection{Rol y articulación L1-L2 en el proceso de alfabetización}

La LSA no solo participa, entonces, como L1, sino en la aproximación a la L2 en una organización didáctica que, en nuestra posición, se implanta en el enfoque de lengua segunda para el español.

La práctica educativa debe ajustar las posibilidades de adquisición de la lengua escrita mediante el interjuego de dos lenguas de naturaleza diferente, y que no coinciden término a término, de las cuales una de ellas (LSA -L1-) no tiene escritura.

En este planteo educativo, desde temprana edad, los niños pueden participar de narraciones a través de inputs textuales en LSA y a partir de allí, generalizar esquemas de los cuentos, por ejemplo, como los niños oyentes lo pueden hacer gracias a las narraciones orales de los adultos. Asimismo, desde pequeños participan de la experiencia de la lectura «en voz alta» en LS (Schleper, 1992). Gracias a ella, pueden acercarse a lo que los textos dicen, también a la literatura al igual que los niños de su edad, y así indagar y preguntar, hipotetizar e imaginar, e incluso dialogar sobre las características de la escritura.

${ }^{9}$ Comparando estudiantes sordos hijos de padres sordos y estudiantes sordos hijos de padres oyentes, Charrow y Fletcher (1974, citados por Marschark 1993) descubrieron que los estudiantes sordos de 17 y 18 años con padres oyentes demuestran un peor desempeño en la prueba de vocabulario TOEFL (Test of English as a Foreign Language) que los estudiantes sordos hijos de padres sordos. El patrón de resultados en esta prueba llevó a los autores a indicar que los estudiantes sordos con padres sordos muestran un desempeño similar al presentado por los estudiantes oyentes que aprenden una segunda lengua (Herrera, 2005).

NORMAS. REVISTA DE ESTUDIOS LINGÜÍITICOS HISPÁNICOS, NÚMERO 3 (AÑO 2013):

http://www.uv.es/normas

(ISSN 2174-7245) 
Existen evidencias acerca de cómo los adultos sordos leen a los niños sordos. Schleper (1997), Lartz y Lestina (1993) describen cómo el papel de la LS evoluciona en relecturas del mismo texto en distintas instancias, desde la narración de la historia basándose en las imágenes, pasando luego por agregar más información de la dicha expresamente en el texto $\mathrm{y}$, finalmente, a leer remitiéndose estrictamente a lo escrito; en este último caso, la actividad consiste en señalar primero la totalidad de la frase que luego será leída en LS.

A la hora de la alfabetización propiamente dicha, al acercar a los textos queriendo descifrar con más exactitud los significados y tener que construir el sistema lingüístico del español, otras intervenciones se hacen imprescindibles y lo serán también cuando los textos escritos presenten temas curriculares en momentos más avanzados de la escolaridad.

La LSA desempeña el lugar de la «oralidad» en el proceso de alfabetización. Participa en el proceso para dialogar, interactuar, preguntar, planificar, en manos del docente para guiar la comprensión y para dar explicaciones.

La propuesta metodológica para el español se estructura, entonces, a partir de la alternancia y simultaneidad, en el mismo período escolar, de exploración libre y también guiada de materiales de lectura, pero su presentación no es siempre casual o anárquica. Tienen lugar centralmente la lengua en uso como principio ordenador, una secuenciación lingüística que permita estabilizar conocimientos lingüísticos, la selección de materiales adecuados y la puesta en acto de variadas estrategias lectoras (como anticipaciones, inferencias, hipótesis, suspensiones), así como la lectura a partir de la cohesión textual; habilidades que deberán ser apropiadas a las distintas situaciones, propósitos y textos. La enseñanza de la lectura requiere, a la vez, del procesamiento sintáctico y no solo del tipo significado-significante.

Se hace foco en el significado y en la forma, sin convertir las clases en «clases de gramática»; el crecimiento de los alumnos irá ampliando las posibilidades de reflexión metalingüística, pero el aspecto central es la recurrencia (distinta de la repetición de lo mismo) y la variedad en los inputs. Este enfoque se ha encontrado también en Berent (1993) y Berent et al. (2007) definido como un programa que repara en el significado y, a la vez, luego se dedica a la instrucción directa de sintaxis y léxico, siendo un enfoque entre los varios posibles en la didáctica de lenguas segundas-extranjeras ${ }^{10}$.

Se configuraría así un trayecto en cuyo transcurso, nos parece, que el lugar de la LSA debe evolucionar para no quedar ligada, a lo largo de la escolaridad, a un rol de traducción

10 Long (1997) describe la enseñanza centrada en la forma (ECF), que incluye diferentes posibilidades: foco en la forma (on forms), foco en el significado (on meaning), foco en ambos (on form). Difiere de la clasificación de Ellis (2001), que distingue foco en la forma (on forms), foco planificado en la forma (se trata de modalidad on form), foco en la forma incidental (modalidad on form). La diferencia que los autores hacen entre el singular y el plural on form/on forms se refiere a la atención primordial al significado y a la forma deducida a partir de él (on form) y al aprendizaje de una forma de la lengua preseleccionada (on forms), con particularidades sobre el tipo de secuenciación didáctica en cada caso (sintetizado de Lucha Cuadros, 2006. Acceso: www.marcoele.com).

NORMAS. REVISTA DE ESTUDIOS LINGÜÍSTICOS HISPÁNICOS, NÚMERO 3 (AÑO 2013):

http://www.uv.es/normas

(ISSN 2174-7245) 
de lo dicho en el texto, de lectura «en voz alta» por parte del profesor, o de expresar el significado siempre en primer lugar en LSA antes de que los alumnos se dirijan por sí mismos a la lectura del texto. Tampoco puede ser una herramienta en la que, en toda ocasión, el niño lee «en voz alta» en LSA, mientras va diciendo al maestro lo que está escrito para demostrar comprensión.

La lectura en voz alta correspondiendo a cada palabra una seña puede verse como una estrategia en etapas iniciales, que muchos niños luego van abandonando. Sin embargo, muchas veces son los docentes quienes sostienen como una lectura término a término (español señado $-\mathrm{ES}^{-11}{ }^{11}$ ), que luego los niños adoptan trayendo confusiones en el procesamiento del significado.

Debemos distinguir, en los casos mencionados, dos situaciones que obedecen a razones distintas. En el primer caso, puede entenderse como un procesamiento término a término mediante el cual el niño encuentra significados en las palabras tratadas aisladamente en una etapa inicial del nivel lector. Pero en estas ocasiones y, desde luego en adelante, es importante guiarlo hacia el procesamiento por grupos de palabras (sintagmas) y en un abordaje discursivo que le permita construir la verosimilitud de la situación que el alumno va organizando a partir de lo que lee; por ello, es necesario que active conocimientos previos, de mundo y del cotexto. Un alumno de una escuela primaria aporta un ejemplo muy ilustrativo respecto de este punto: cuando leía la oración Sarmiento fue maestro, y a cada palabra correspondía una seña, indicaba el verbo «fue» como verbo IR. Seguidamente, se le propuso que releyera desde la oración anterior en silencio. Al solicitársele posteriormente el significado, expresó PASADO SARMIENTO MAESTRO, es decir que el primer modo de leer obstaculizaba la comprensión que, en silencio, evidenciaba. En el segundo caso, la lectura «en voz alta» del docente debería consistir en ir señalando oraciones, sintagmas ${ }^{12}$ y/o párrafos en primer lugar en silencio, para luego expresar el significado en LSA y no, en ES.

Sin embargo, queremos distinguir algún uso productivo del ES cuando puede emplearse si se quiere recalcar el orden de los significantes o el lugar en el texto en que algo está dicho y/o comparar estructuras entre las lenguas, en cuyo caso, estaríamos reflexionando sobre las lenguas. Por ejemplo, para expresar frases como el auto de Andrés, la casa de Sofía, es diferente hacerlo en LSA con su estructuración propia, de querer enfatizar el orden de los constituyentes en español y enfatizar que la preposición de, en estos ejemplos, designa pertenencia ${ }^{13}$. En estas situaciones, podríamos aceptar observar el orden gramatical español mediante señas y/o alfabeto dactilológico (por ejemplo, diciendo

\footnotetext{
${ }^{11}$ Español señado es el empleo simultáneo de la lengua oral y señas de la lengua de señas. Tales señas se incluyen en la organización sintáctica de la lengua oral.

${ }^{12}$ El sintagma es una unidad constituida por una o varias palabras que desempeñan una función unitaria dentro de la oración.

${ }^{13}$ En otros casos, la preposición de introduce material (por ejemplo, de madera) o refiere al tiempo y espacio (de 8 a 12 horas; de norte a sur).
} 
D-E S-O-F-Í-A o D-E SOFIA ${ }^{14}$ ) y por ello deletrear la preposición, alternativa inadmisible en la estructuración propia de la LSA. En este sentido, investigaciones desarrolladas por Gioia, Johnston y Cooper (2001) creen que el inglés señado es un puente útil para conectar el ASL y el inglés escrito, en tanto «juega solamente un breve papel de mediación».

Un diálogo de características metalingüísticas y metacognitivas entre profesores y alumnos es muy difícil de verificar en modelos estrictamente orales, en los que muchas veces la ausencia de lengua fluida en común entre los participantes de la clase, en virtud del nivel alcanzado en la oralidad ${ }^{15}$, impide estas tareas. En estos casos, se suele acudir a soluciones no lingüísticas (la dramatización, el empleo de imágenes), propias de formas iniciales de la comunicación, o muchas veces insuficientes, y que pierden la riqueza de todo el conocimiento que se desarrolla entre las lenguas, como ocurre en todo bi(pluri)lingüismo.

\subsubsection{El análisis del código}

Para construir conocimiento léxico, no solamente deben abordarse las palabras a nivel del significado, sino también mediante un trabajo de análisis de los significantes, es decir de la constitución interna de las unidades léxicas.

La adquisición léxica que, en principio, se adquiriría por vía visual y mediante palabra completa, supone a la vez un proceso de construcción de las regularidades del sistema; por lo tanto, es parte del proceso de apropiación de la lengua escrita establecer relaciones previsibles y económicas en el sistema (Camps et al., 1999) que los niños sordos no pueden cimentar centralmente en la conversión grafema-fonema, relaciones que sí ocupan un lugar preponderante en oyentes en español, dado que las relaciones grafemafonema claramente se basan en la audición y en léxico auditivo disponibles.

Es necesario que la propuesta de enseñanza supere esta restricción que experimentan los alumnos sordos y pueda ayudarlos a construir relaciones sistemáticas. Relaciones sistemáticas estarían dadas, en principio, por la vinculación entre las unidades significativas de las palabras (morfemas) y la ortografía, unidades pasibles de ser percibidas visualmente, lo cual, al mismo tiempo, es indivisible de la adquisición del léxico, ya que el conocimiento morfológico permite especialmente dar significado a palabras nuevas en el conjunto de lo que el texto dice, ya que el procesamiento debe superar el nivel de la palabra. Luego, podrán abordarse progresivamente $\mathrm{y}$, a los fines del recuerdo del léxico, las comparaciones

${ }^{14}$ Las palabras completas en mayúsculas significan que son expresadas en LS. Cuando sus letras aparecen separadas por guiones, significa el empleo de castellano señado.

15 Reflexionar sobre la lengua, plantear actividades de prelectura o poslectura supone un nivel lingüístico superior al contenido que se está aprendiendo y resultan inalcanzables porque el nivel de desarrollo alcanzado en la lengua no lo posibilita.

NORMAS. REVISTA DE ESTUdIOS LINGÜÍSTICOS HISPÁNICOS, NÚMERO 3 (AÑO 2013):

http://www.uv.es/normas

(ISSN 2174-7245) 
entre palabras basadas en las sílabas y en grafemas para favorecer diversos modos de almacenamiento y recuperación de las palabras.

Debemos agregar que existen evidencias acerca de que los lectores sordos utilizan una variedad de estrategias de decodificación. En el análisis de los significantes, también operan la LSA y en especial, el alfabeto dactilológico. Algunos autores señalan «estrategias sordas» usadas por padres y profesores sordos que conectan la lengua de señas con la lengua escrita. Entre ellas, chaining: una palabra es deletreada, luego se señala la palabra escrita y luego es deletreada otra vez (Erting et al. 2000; Padden y Ramsey, 1998, 2000); sandwiching: la palabra es signada, luego se muestra la palabra escrita para luego ser signada otra vez (Blumenthal- Kelly, 1995). Otra posibilidad apreciada es vincular las lenguas uniendo elementos específicos del ASL e inglés impreso que apoyan la lectura (Padden y Ramsey, 1998). Se trataría de estrategias complementarias disponibles en momentos alternativos.

En el trabajo con los significantes, entonces, es necesario admitir un concepto más ampliado de fonología (Alegría, 2004). Como vemos, tanto para construir la representación interna de las palabras como en su recuperación para escribirlas, se observa la utilización del alfabeto dactilológico junto con otras estrategias de naturaleza visual (articulación en lengua oral, lectura labial, vinculación con LSA).

Entre todas ellas, la preeminencia asignada a la dactilología fue especialmente observada por Hirsh-Pasek (1997); Chamberlain y Mayberry (2000) y su eficacia estaría en tener una estructura alfabética que la hace apta como sistema de anotación manual de escritura (Domínguez y Alonso, 2004). Según Padden (1993), los niños sordos vinculan palabras escritas con letras del alfabeto, en lugar de vincular palabras habladas con palabras escritas. Específicamente, Musselman (2000) señala que el uso de la codificación fonológica por los lectores sordos puede ser un resultado del aprendizaje de leer y no una condición antecedente. En nuestras investigaciones es importante señalar que se percibe frecuentemente cómo a partir de la escritura y de la LSA, puede significarse una señal acústica, que hasta ese momento, estaba desprovista de significado.

\subsubsection{La escritura autónoma}

Muchas veces, se introduce a los niños sordos en situaciones de escritura para alguna ocasión particular y contingente sin los conocimientos necesarios, generando un rompecabezas que luego es resuelto por una participación docente en exceso, sin que puedan estabilizarse a posteriori conocimientos que permitan a los alumnos afrontar situaciones nuevas con mayor autonomía cada vez; se genera un «escribir en LSA», por medio de lo cual, los alumnos van plasmando sus ideas en papel con el orden gramatical LSA, y seguidamente el docente es el ordenador de las mismas «transcribiendo» al español y se cierra la actividad.

NORMAS. REVISTA DE ESTUdIOS LINGÜÍSTICOS HISPÁNICOS, NÚMERO 3 (AÑO 2013):

http://www.uv.es/normas

(ISSN 2174-7245) 
Encontramos una dificultad importante en plantear a los niños escribir a partir de su producción en LSA en una suerte de transferencia directa. El proceso de escritura, si bien puede comenzar en LSA con un momento de exposición de sus ideas, de discusión acerca de la forma que adoptará el texto, sobre los saberes lingüísticos necesarios, etc., tiene que concretarse a partir de la planificación en español, disponiendo de la experiencia previa de abordaje de textos como los que necesitan escribirse, teniendo a su alcance modelos y activando tanto aspectos discursivos, del tema en sí, como específicamente lingüísticos de la L2. El momento de escritura de borradores y revisiones progresivas desde un nivel macro (tipo de género, propósito de escritura, destinatario, coherencia general) a lo micro (aspectos estrictamente lingüísticos desde sintaxis a léxico y ortografía, colocaciones, puntuación) es un trabajo sumamente necesario y fructífero.

A través de más de veinte años de investigación y docencia, observamos cómo la planificación textual favorece que, en el término de pocos meses, los alumnos pasen de escribir oraciones confusas en español a presentar, como primer logro, escrituras que componen en primer lugar textos, progresando en la coherencia e incrementando las posibilidades de escribir más cantidad y con mayor calidad. Especialmente es notorio cómo pueden reflexionar sobre sus escrituras y producir avances en forma constante.

Todo ello ha sido conseguido al delimitar la participación de las lenguas: LSA para el intercambio de ideas, español para planificar español sobre la organización discursiva, léxica y sintáctica. Aunque en la revisión de los textos producidos se advierta interferencia lingüística de la LSA, es muy productivo el análisis de lo que se formuló en forma inadecuada $^{16}$ si se coteja con textos ya conocidos y con información lingüística que se haya sistematizado previamente.

Los fallos de los estudiantes durante la producción escrita informan acerca del proceso de interlengua que está ocurriendo. Como detalla Fernández López (1989), el error es un paso necesario, ya que aporta evidencia acerca de los procesos de adquisición e indica si el alumno va progresando hacia la L2.

Para Rigamonti (1998), el error es una hipótesis incorrecta del funcionamiento de la lengua. La autora plantea de modo muy interesante que las conjeturas que realiza el alumno están sujetas a comprobación continua mediante la observación de lo producido por otros y por él mismo. Si infiere que alguna de sus hipótesis no corresponde al uso, la produce hasta encontrar la forma correcta y añade que, en el período de comprobación, produce tanto la forma correcta como la incorrecta por igual. Sugiere que ese es el momento propicio para la

${ }^{16}$ Interlengua: «Las peculiaridades del proceso de adquisición de una L2/LE ponen de manifiesto una serie de estadios o fases a través de los cuales el aprendiz va construyendo de manera global un sistema aproximativo a la lengua objeto de aprendizaje, que constituye por sí mismo un sistema lingüístico en estado continuo de crecimiento y fluctuación, llamado interlengua» (Torras, 1994). Es importante precisar que error se atribuye a un conocimiento inadecuado de alguna forma de la lengua meta, a diferencia de la equivocación, la cual se debe a situaciones independientes del conocimiento de la lengua (distracción, cansancio).

NORMAS. REVISTA DE ESTUDIOS LINGÜÍSTICOS HISPÁNICOS, NÚMERO 3 (AÑO 2013):

http://www.uv.es/normas

(ISSN 2174-7245) 
intervención docente. Es central la claridad acerca de cuándo intervenir, cómo hacerlo y qué corregir, yendo de lo comunicativo (adecuación pragmática, discursiva) a lo lingüístico (morfosintaxis, selección y colocación léxica, ortografía, acentuación, puntuación).

La valoración de los errores cometidos por los niños y jóvenes sordos en la producción escrita es muy diferente si se realiza en comparación con el grupo de estudiantes extranjeros de una lengua mayoritaria, en lugar de ser analizada en relación con la escritura producida por sujetos oyentes. Apreciados desde el mismo punto de vista con el que se consideran los errores manifestados por alumnos extranjeros, no solo podrá verse la similitud entre ellos, sino que, además, estos proporcionan más información sobre la adquisición de aprendizajes que sobre las dificultades que, en general, se les atribuyen a los sordos solo por el hecho de serlo.

El concepto de interlengua, asimismo, explica que la evolución de los alumnos en una lengua segunda no procede por suma de contenidos; se producen saltos en determinados momentos, desajustes y nuevas integraciones. Permite al docente conocer las necesidades y evaluar los progresos del alumno.

En la observación de situaciones de escritura, análogamente que en la lectura, se observa la alternancia de lenguas y diferentes modos de codificación de naturaleza visual, sin corresponder la utilización de la LSA con menor nivel auditivo y oral. Aún con muy buen desarrollo de la oralidad, los niños demuestran la recuperación de algunas palabras mediante el procesamiento fonológico, a través del alfabeto dactilológico (propio o recurriendo a otro compañero o profesor). Belles (2000) asegura que este sistema faculta a los niños de autonomía para escribir a partir de la lengua de señas. Mayer, Akamatsu y Stewart (2002) destacan también el uso de la lengua mayoritaria signada como puente entre ambas lenguas, fundamentalmente al escribir. Asimismo, los sordos demuestran emplear, con el mismo fin de recuperación de formas escritas, la lectura labial, la articulación oral (con y sin voz) como lo hacen durante la lectura. Todas estas estrategias que hemos mencionado en este apartado son empleadas en forma secuencial, simultánea o alguna de ellas preferencialmente por los distintos escritores sordos (Lapenda, 1992; 1999).

\section{CONCLUSIONES}

La educación de los estudiantes sordos tiene que concretar una nueva construcción en lugar de configurarse como un proceso similar al de los sujetos oyentes, pero incompleto, entendido como desfasado, más lento o asumiendo que tendrá inevitablemente déficits que los alumnos deben compensar o que, inclusive jamás podrán lograr.

Precisamente en este artículo se da cuenta de que un vínculo metacognitivometalingüístico entre LSA-español, así como la transferencia de conocimientos específicamente lingüísticos entre ellos, reportan notables beneficios en el proceso de alfabetización siempre y cuando, además, haya una propuesta didáctica sólida para el 
español. También dan cuenta del desarrollo de una competencia para aprender a aprender que conducirá al acceso a variedad de lenguas sin obstáculos.

En este sentido, es relevante destacar que son los mismos niños quienes demuestran una integración de posibilidades, junto con la elección de aquellas que consideran más propicias según la situación, cuando a partir de la LS significan la información auditiva obtenida gracias a su implante coclear; cuando por la escritura dan sentido a la escucha; al optimizar su producción oral por la propia lectura en voz alta; o aún con importante desarrollo oral y auditivo, al leer palabras desconocidas, analizándolas con el alfabeto dactilológico, lo cual inclusive les permite luego leerlas en voz alta. También así se observa la alternancia de lenguas (LSA-español) con distintos fines durante las clases.

La productividad de estrategias diversas es fruto de la enseñanza, con lo cual puede concluirse que metodologías adecuadas conducen a muy buenos resultados, tanto en lectura como en escritura.

\section{REFERENCIAS BIBLIOGRÁFICAS}

AlegríA, Jesús (2004): «Deafness and Reading», en Nunes, Terezinha y Peter Bryant, eds., Handbook of Children's Literacy, Dordrecht, Kluwer Academic Publishers, 459-492.

ALLEN, Thomas (1986): «Patterns of academic achievements among hearingimpaired Students: 1974-1983», en Schildroth, Arthur N. y Michael A. Karchmer, eds., Deaf children in America, Austin, Texas, Pro-ed., 217-248.

ANDREWS, Jean F. y Jana M. MASON (1991): «Strategy usage among deaf and hearing readers». Exceptional Children, 57, 6, 536-545.

ANDREWS, Jean et alii (1997): «What's Up, Billy Jo? Deaf Children and BilingualBicultural Instruction in East Central Texas», American Annals of the Deaf (Washington, Gallaudet University Press), 142, 1, 16- 25.

BAKER, Colin (1993): Fundamentos de Educación bilingüe y bilingüismo, Madrid, Ediciones Cátedra.

BELles GuitART, Rosa (2000): «La construcció interactiva de l'escriptura en nens i nenes sords petits mitjancant la dactilología», Support, Revista Catalana d'Educació Especial i Atenció a la Diversitat, 4, 1, 38-50.

BERENT, Gerald P. (1993): «Improvements in the English syntax of deaf college students», American Annals of the Deaf, 138, 1, 55-61.

BERENT, Gerald P. (1996): «The acquisition of English syntax by deaf learners», en Ritchie, William C. y Tej K. Bhatia, eds., Handbook of Second Language Acquisition, San Diego, CA: Academic Press, 469-506. 
BERENT, Gerald P. y William ClyMER (2007): «Professional Development for Educators Teaching English for Academic Purposes to Deaf Students of English as Foreign Language», en paper, Nueva York, Pen International, NITD.

BlumentHAL-Kelly, Arlene (1995): «Fingerspelling interaction: A set of deaf parents and their deaf daughter», en Lucas, Ceil (ed.), Sociolinguistics in deaf communities, Washington D.C., Gallaudet University Press, 62-73.

CANAlis, Ángel Huguet e Ignasi Vila MEndiburu (1997): «Nuevas aportaciones a la Hipótesis de Interdependencia Lingüística en escolares bilingües», Infancia y aprendizaje, 79, 21-34.

Chamberlain, Charlene; Jill MORford y Rachel I. MAYBerRY, eds., (2000): Language Acquisition by Eye, Mahwah, NJ: Lawrence Erlbaum and Associates, 881-938.

Charrow, Veda R. (1981): «The written English of Deaf Adolescents», en Farr Whiteman, M., ed., Writing: The Nature, Development, and Teaching of Written Communication, volumen 1, Hillsdale, NJ: Lawrence Erlbaum Ass., 179-187.

Charrow, Veda R. y John D. FleTCHER. (1974): «English as a second language of Deaf children», Developmental Psychology, 10, 463-470.

COLLIER Virginia P. (1995): Promoting academic success for ESL students: Understanding second language acquisition for school, Elizabeth, NJ: New Jersey Teachers of English to Speakers of Other Languages-Bilingual Educators.

COOPER, Robert L. y Joseph RosEnSTEIN (1996): «Language acquisition of the deaf children», Volta Review, 68, 58-67.

CuMmins, Jim (1979): «Linguistic Interdependence and the Educational Development of Bilingual Children», Review of Educational Research, 49, 221-251.

CUMMINS, Jim (2004): «La interdependencia en el aprendizaje de idiomas» Conferencia en San Sebastián len línea]: <http://www.aprendemas.com/Noticias/html/N525_F26072004.HTML>. [Consulta: abril 2012].

Cummins, J. (2006, October 27): «The relationship between ASL proficiency and English academic development: A review of the research», paper presented at the Workshop Challenges, Opportunities, and Choices in Educating Minority Group Students, Hamar University College, Norway.

DAVIES, Shawn (1994): «Attributes for success: Attitudes and practices that facilitate the transition towards bilingualism in the education of deaf children», en Ahlgren, Inger y Kenneth. Hyltenstam, eds., Bilingualism in deaf education, Hamburg (Germany), Signum, 103-121.

Delmastro, Ana Lucía (2005): «Constructivismo y Enseñanza de Lenguas Extranjeras», Tesis Doctoral, Facultad de Humanidades y Educación, Luz. Maracaibo.

DíAZ, Estela (2005): El sujeto sordo en el lenguaje, Buenos Aires, Ediciones I Rojo. 
Domínguez Gutierres, Ana Belén y Pilar Alonso BaiXeras (2004): La educación de los alumnos sordos hoy. Perspectivas y respuestas educativas, Málaga, Ediciones Aljibe.

Domínguez GuTIÉRRES, Ana Belén (1995): El aprendizaje de la lectura en los niños sordos. Una revisión del estado actual de la investigación, Salamanca Ed., Universidad Pontificia de Salamanca.

Domínguez GutiÉRRES, Ana Belén (1999): «Lenguaje escrito y sordera: ¿Sobre qué cuestiones es importante reflexionar? », en Domínguez Gutiérres, Ana Belén y Carmela Velasco Alonso, coords., Lenguaje escrito y sordera. Enfoques teóricos y derivaciones prácticas, (Salamanca, Universidad Pontificia de Salamanca), 169-175.

DuRgunoglu, Aydin Yucesan (2002): «Cross-linguistic Transfer in Literacy Development and Implications for Language Learners, Annals of Dyslexia» [en línea]: <http://www.findarticles.com/p/articles/mi_qa3809/is_200201/ai_n9067682>. [Consulta: 06/11/ 2011].

ELLIS, Rod (1994): The Study of Second Language Acquisition, Oxford University Press.

Erting, Carol; Carlene Thumann-Prezioso y Barbara Benedict (2000): «Bilingualism in a Deaf family: Fingerspelling in early childhood», en Spencer, Patricia E., Carol Erting, y Marc Marschark, eds., The deaf child in the family and at school, Mahwah, NJ, Erlbaum, 41- 54.

FERnÁNDEZ LóPEZ, María Sonsoles (1989): «Corregir y evaluar desde una perspectiva comunicativa», Cable, 4, 30-35.

FERnÁndeZ ViAdER, María Pilar y Esther PERTUSA (1996): «Reflexiones sobre la escritura y la alfabetización en niños sordos», Revista Logopedia, Fonología y Audiología, $16,2,79-85$.

GASS, Susan (1984): «A review of interlanguage syntax: Language transfer and language universals», Language Learning, 34, 2, 115-131.

GASS, Susan y Larry SELINKER (2001): Secong Language Acquisition. An Introductory Course, Mahwah, NJ: Lawrence Erlbaum Associates.

GutiÉRrez GÁCERES, Rafaela (2004): Cómo escriben los sordos, Málaga, El Aljibe.

HAMilton, Harley y Thomas G. HolzMAN (1989): «Linguistic encoding in shortterm memory as a function of stimulus type», Memory y Cognition, 17, 5, 541-550.

HEILING, Kerstin (1999): «La lectura y la escritura en los niños sordos en contextos bilingües», en Domínguez, Ana Belén y Carmela Velasco Alonso, coords., Lenguaje escrito y sordera. Enfoques Teóricos y Derivaciones Prácticas, Salamanca, Universidad Pontificia de Salamanca, 179-182.

HERRERA, Valeria (2003): «Bilingüismo y lectura de las personas sordas» [en línea]: <santiagoapostol.k12.cl/core/view/5654>. [Consulta: 10/12/2012].

HERRERA, Valeria (2005): «Habilidad lingüística y fracaso lector en los estudiantes sordos», Estudios Pedagógicos, volumen, 31, 2, 121-135. 
HIRSH-PASEK, Kathy (1987): «The metalinguistic of fingerspelling: An alternate way to increase reading vocabulary in congenitally deaf readers», Reading research Quarterly, 22, 4, 455-473.

HofFMEISTER, Robert (2002): «A Piece of the Puzzle: ASL and Reading Comprehension in Deaf Children», en Spencer, Patricia E; Carol Erting y Marc Marschark, eds., The Deaf Child in the Family and at School, 143-163.

GIBSON, Heather; Anita SMALl y David MASON (1997): «Deaf bilingual Bicultural Education», en Jim Cummins y D. Corson, eds., Encyclopedia of Language and Education. Bilingual Education, volumen 5, Kluwer Academic Publishers, 231-240.

GIOIA, Barbara et alii (2001): «Documenting and developing literacy in deaf children», Literacy Teaching and Learning, 6, 1, 1-22.

KInG, Cynthia M. y Stephen Quigley (1985): Reading and Deafness, San Diego, College Hill Press.

KERPER-MORA, Jill (2000): «Metalinguistic Transfer in Spanish/English Biliteracy» [en línea]: <http://coe.sdsu.edu/people/jmora/MoraModules/MetalingTransfer.htm>. [Consulta: noviembre 2012].

Krashen, Stephen (1982): Principles and Practices in Second Language Acquisition, Oxford, Pergamon Press.

KRASHEN, Stephen (1983): «Newmark's ignorance hypothesis and current second language acquisition theory», en Gass, Susan y Larry Selinker, eds., Language Transfer in Language Learning (Rowley, Mass: Newbury House), 135- 153.

KRASHEN, Stephen (1989): «We acquire vocabulary and spelling by reading: Additional evidence for the input hypothesis», Modern Language Journal, 73, 440-464.

KRASHEN, Stephen (1996): Under Attack: The Case Against Bilingual Education, Culver City, Language Education Associates.

KRASHEN, Stephen (2006): «Bilingual Education Accelerates English Language Development» [en línea]: <http://www.sdkrashen.com/index.php?cat=5/>. [Consulta: 03/02/2013].

KunTZE, Marlon (1998): «Literacy and Deaf Children: The Language Question», Topics in language disorders, 18, 4, 1-5.

LAPENDA, María Eugenia. (2005): «Enseñar a leer y escribir niños sordos». Novedades Educativas, 174 (Buenos Aires. Novedades Educativas Ed.), 28-33.

LAPENDA, María Eugenia y RuSELl, Gabriela Susana (2007): «Circular Técnica N³. Educación intercultural bilinguie: LSA-español. Prácticas del lenguaje», Dirección de Educación Especial, DGCyE, Provincia de Buenos Aires.

LANGSTON, Cynthia A. y Madeline L. MAXWELl (1988): «Holistic Judgement of Texts by Deaf and ELS Students», Sign Language Studies, 60, 295-312.

LARTZ, Maribeth y Jason LESTINA (1993): «Strategies Deaf mothers use when reading to their young Deaf or Hard of Hearing children», American Annals of the Deaf, 140 (Washington, Gallaudet University Press), 358-362. 
Lucha CuAdros, Rosa M. (2006): «Por qué enseñar a escribir usando técnicas focus on form» [en línea]: 〈www.marcoele.com>.

Massone, María Ignacia; Marina Simón y Juan Carlos DruetTA (2003): «Una aproximación a la lengua escrita», Arquitectura de la escuela de sordos, Libros en Red.

MARSCharK, Marc; Harry G. LANG y John A. Albertini (2002): Educating Deaf Studies, Nueva York, Oxford University Press.

MARSCHARCK, Marc (1997): «Learning to read and write», en Marscharck, Marc, ed, Psychological development of deaf children, New York, Oxford University Press.

MAYER, Connie y Tane AKAMATSU (1999): «Bilingual Bicultural Models of Literacy Education for Deaf Students: Considering the claims», Journal of Deaf Studies and Deaf Education, 4, 1, 1-8.

Mies Burrull, María Àngels (1992): «El léxico escrito en la comprensión lectora: estudio de un grupo de alumnos sordos profundos», Revista de Logopedia. Foniatría y Audiología, 22, 4, 203 - 212.

Mitchell, Rosamond y Florence MYLES (2004): Second Language Learning Theories, Londres, Arnold Publishers.

MuRPHY, Shirin (2002): «Second Language Transfer During Third Language Acquisition»: <http://www.tc.columbia.edu/academic/tesol/Webjournal/Murphy.pdf>. [Consulta: 03/02/2013].

Musselman, Carol (2000): «How do children who can't hear learn to read an alphabetic script? A review of the literature on reading and deafness», Journal of Deaf Studies and Deaf Education, 5 (New York, Oxford University Press), 9-31.

NeESE BAILES, Cynthia et alii (2009): «Language and Literacy Acquisition through parental Mediation in American Sign Language», Sign Language Studies, 9, 4, (Washington. Gallaudet University Press), 417-456.

ODLIN, Terence (1989): Language Transfer. Cross-linguistic Influence in Language Learning, Cambridge, Cambridge University Press.

PADDEn, Carol y Tom Humphries (1988): Deaf in America, Voices from a Culture, Cambridge, Mass, Harvard University Press.

PADDEN, Carol y Claire RAMSEY (2000): «American Sign Language and Reading Ability in Deaf Children», en Chamberlain, Charlene; Jill P. Morford y Rachel I. MayBerry, eds., Language Acquisition by Eye, Mahwah, NJ: Lawrence Erlbaum Ass.

PADDEN, Carol (1993): «Lessons to be learned from the young deaf orthographer», Linguistics and Education, 5, Kamberelis, George A. Editor, 71- 86.

PAUL, Peter V. (1998a): Literacy and Deafness: the development of reading, writing and literate thought, Boston, Allyn and Bacon.

PAUL, Peter V. (1998b): «Reading for students with hearing impairments: Research review and implications», The Volta Review, 99, 2, 73-88.

Pertusa Venteo, Esther y María Pilar Fernández Viader (2004): «La lengua escrita en el niño sordo: la escritura», en Fernández Viader, María Pilar y Esther Pertusa 
Venteo, coords., El valor de la mirada: sordera y educación, Barcelona, Edicions Universitat Barcelona.

PETITTO, Laura Ann (2000): «The Acquisition of Natural Signed Languages: Lessons in the Nature of Human Language and Its Biological Foundations», en Chamberlain, Charlene; Jill P. Morford y Rachel I. MayBerry, eds., Language Acquisition by Eye, Mahwah, NJ: Lawrence Erlbaum Ass., 881-938

PETITTO, Laura Ann y Siobhan HolowKA (2002): «Evaluating attributions of delay and confusion in young bilinguals: Special insights from infants acquiring a signed and spoken language», Sign Language Studies, 3, 1, 4-32.

Quigley, Stephen P.; Ronnie WiLbUR y Dale S. MonTANelli (1976): «Complement structures in the language of deaf students», Journal of Speech and Hearing Research, 17, 699-713.

RIGAMONTI, Daniela (1999): «La corrección del error en la producción escrita: el caso de estudiantes italófonos de E/LE», Revista Carabela, 46, Madrid, SGEL, 137-151.

RICHARDS, Jack; John Platt y Heidi PlatT (1997): Diccionario de lingüística aplicada y enseñanza lenguas, Barcelona, Ariel.

Ruiz Bolívar, Carlos (2002): «Mediación de Estrategias Metacognitivas en Tareas Divergentes y Transferencia Recíproca», en Investigación y Postgrado, volumen 17, 2, 53 82 [en línea]: 〈http://www.scielo.org.ve>. [Consulta: 19/2/2012].

RODRÍGUEZ ORTíz, Isabel Reyes y Joaquín MORA RoCHE (2008): «The Efficiency of Information Transmission of Sign and Spoken Language», en American Annals of the Deaf, 152, 5 (Washington: Gallaudet University Press), 480- 494.

RodrígueZ SANTOS, José Miguel; Javier GARCíA ORZA y Santiago TORRES MONREAL (1997): «El uso de estrategias sintácticas en sujetos sordos», Revista de Psicología del Lenguaje, 2, 117-135.

SALAZAR, Leonor (2006): «Interdependencia lingüística, transferencia y enseñanzaaprendizaje de lenguas extranjeras», Laurus, volumen 12, 45-72 (Caracas).

SELINKER, Larry (1972): «Interlanguage», International Review of Applied Linguistics, 10, 3, 209- 231 (editado por Peter Jordens, Eric Kellerman y Leah Roberts).

SCHLEPER, David R. (1997): Reading to Deaf Children: Learning from Deaf Adults (Leyéndoles a niños sordos: Aprendiendo de adultos sordos), Washington D.C., Gallaudet University Press.

Schlesinger. Hilde S. y Kathryn P. Meadow (1972): Sound and Sign: Childhood Deafness and Mental Health, Berkeley, CA, University of California Press.

SIEGEL, Lawrence (2000): «The Argument for a Constitutional Right to Communication and Language», Journal of Deaf Studies and Deaf Education, 7, 3, New York, Oxford University Press, 258-266.

Singleton, Jenny L. y Dianne D. MoRgAN (2006): «Natural Sign Language Acquisition Within the Social Context of the Classroom», en Schick, Brenda; Marc 
Marschark y Patricia E. Spencer, eds., Advances in the Sign Language Development of Deaf Children, 18, 4, Nueva York, Oxford University Pres, 16-29.

STOCKSETH DANZAK, Robin (2002): «Comprensión de la sintaxis española en los sordos chilenos», Revista Signos, 35, 51-52, 271-290.

STRONG, Michael y Philip M. PRINZ (2000): «Is American Sign Language Skill Related to English Literacy? », en Chamberlain, Charlene; Jill P. Morford y Rachel I. MayBerry, eds., Language Acquisition by Eye, Mahwah, NJ: Lawrence Erlbaum Ass.

Svartholm, Kristina (1993): «Bilingual Education for Deaf people in Sweden», Sign Language Studies, 81, 291-331.

TORRAS, M. Rosa (1994): «La interlengua en los primeros estadios de aprendizaje de una lengua extranjera (inglés)», CL y E: Comunicación, lenguaje y educación, 24, 49-62.

TORREGO, Esther (1998): «Temas de gramática y cognición: Primeras y segundas lenguas», en Catalá, Natalia; José A Díez Calzada y José E. García-Albea, coords., El lenguaje y la mente humana, Barcelona, Ariel.

TORRES MOnREAL, Santiago y Rafael SANTANA HERnÁndeZ (2005): «Reading levels of Spanish Deaf Students», American Annals of the Deaf, 150, 4, 379-387.

TrAXler, Carol (2000): «The Stanford Achievement Test, National norming and performance standards for deaf and hard of hearing students», Journal of Deaf Studies and Deaf Education, 5, 337-348, 9. ${ }^{\text {a }}$ ed.

VAlmaseda BAANZATEGui, Marian y Lourdes GómeZ MonTERde (1999): «Formar lectores: Buscando caminos con los alumnos sordos», en Domínguez Gutiérrez, Ana Belén y Carmela Velasco Alonso, coords., Lenguaje escrito y sordera. Enfoques teóricos y derivaciones prácticas, Salamanca, Universidad Pontificia de Salamanca.

VIROLE, Benoit (2003): «Los implantes cocleares en el niño y la utilización temprana de la lengua de señas» [en línea]: 〈www.benoitvirole.com>. [Consulta: 06/03/2012].

VOLTERRA, Virginia et alii (1979): «First words in language and action: A qualitative look», en Bates, Elizabeth, The emergence of symbols: cognition and communication in infancy, New York, Academic Press, 141-222.

VOLTERRA, Virginia (1981): «Gestures signs and words at two years: When does communication become language?», Sign Language Studies, 33, 351-362.

VOLTERRA, Virginia et alii (1990): Gesture and the emergence and development of language, ISTC, CNR.

WAUTERS, Loes et alii (2001): «Sign Facilitation in Word Recognition», The Journal of Special Education, 35, 1, 31-40.

WILBUR, Ronnie (2000): «The Use of ASL to Support the Development of English Literacy», Journal of Deaf Studies and Deaf Education, 5, 1, 81-104.

WIDMAYER, Sharon (2000): «Schema Theory: an introduction». Citado por SALAZAR, Leonor (2006): «Interdependencia lingüística, transferencia y enseñanza-aprendizaje de lenguas extranjeras», Laurus, volumen 12, 45-72. 
